\title{
On 1-regular ordinary differential operators
}

\author{
by GrZEgORZ LYSIK (Warszawa) \\ To the memory of Bogdan Ziemian, \\ my great friend and adviser
}

\begin{abstract}
Solutions to singular linear ordinary differential equations with analytic coefficients are found in the form of Laplace type integrals.
\end{abstract}

Introduction. Let

$$
P\left(x, \frac{d}{d x}\right)=\sum_{i=0}^{n} a^{i}(x) \frac{d^{i}}{d x^{i}}
$$

be a linear differential operator of order $n \in \mathbb{N}$ with coefficients $a^{i}(x)=$ $\sum_{j=0}^{\infty} a_{j}^{i} x^{j}$ convergent for $|x|<r, i=0, \ldots, n-1$, and $a^{n}(x)=x^{m}$ with some $m \in \mathbb{N}_{0}$. Let $\kappa_{P}$ be the Katz invariant for $P$, i.e. the smallest $\kappa \in \mathbb{R}$ such that there are no points of $\mathcal{N}_{P}$ below the line $\left\{(i, j) \in \mathbb{N}_{0} \times \mathbb{Z}: j=\kappa(i-n)+m-n\right\}$ where

$$
\mathcal{N}_{P}=\left\{(i, j) \in \mathbb{N}_{0} \times \mathbb{Z}: a_{i+j}^{i} \neq 0\right\}
$$

is the Newton diagram for $P$. If $\kappa_{P} \leq 0$ then zero is a regular or regular singular point for $P$, and the well known Fuchs theorem states that the fundamental system of solutions of $P u=0$ consists of convergent series of Taylor type, whose coefficients can be easily determined (cf. [CL]). On the other hand, in the case $\kappa_{P}>0$, zero is an irregular singular point for $P$, and there exist power series solutions to $P u=0$ but they need not be convergent. During the last several years a special method called multisummability was worked out to deal with divergent solutions of differential equations. By this method, starting from a formal power series solution, one constructs a holomorphic solution in a sector in $\widetilde{\mathbb{C} \backslash\{0\}}$ having the formal one as its

2000 Mathematics Subject Classification: 34A20, 34A30 44A15.

Key words and phrases: singular differential equations, Laplace integrals, Mellin transformation. 
asymptotic expansion (cf. [B], [E], [M]). Unfortunately, the method cannot be applied directly to the study of the Cauchy problem. We shall describe how the Cauchy problem can be treated by a method based on the Mellin transformation. We shall concentrate on the study of the Cauchy problem for the homogeneous equation $P u=0$ where $P$ is a 1-regular operator, i.e. an operator with $\kappa_{P} \leq 1$. Observe that any operator $P$ with $\kappa_{P}>0$ can be reduced to a 1-regular operator $\widetilde{P}$ by the change of variable $\widetilde{x}=x^{\kappa_{P}}$. The coefficients of $\widetilde{P}$ are analytic functions in the variable $\widetilde{x}^{1 / \kappa_{P}}$, but this should not cause any essential difficulties.

Our method of treatment of the Cauchy problem for $P u=0$ with the Cauchy data at a non-singular point $0<t<r$ can be described as follows. Firstly, we note that any 1 -regular operator $P$ given by (1) with $a^{n}(x)=x^{2 n}$ can be written in the form

$$
P\left(x, \frac{d}{d x}\right)=Q\left(x^{2} \frac{d}{d x}\right)+\sum_{i=0}^{n-1} g^{i}(x)\left(x^{2} \frac{d}{d x}\right)^{i},
$$

where $Q$ is a polynomial of degree $n$ and $g^{i}, i=0, \ldots, n-1$, are functions analytic in the disc $B(r)$ and vanishing at zero. Next, after the change of variable $s(x)=\exp \{1 / t-1 / x\}$ the original Cauchy problem is transformed into the one for the equation $R(s, s d / d s) w=0$ with the Cauchy data at 1 , where

$$
R\left(s, s \frac{d}{d s}\right)=Q\left(s \frac{d}{d s}\right)+\sum_{i=0}^{n-1} \widetilde{g}^{i}(s)\left(s \frac{d}{d s}\right)^{i}
$$

and $\widetilde{g}^{i}(s)=g^{i}\left((1 / t-\log s)^{-1}\right), i=0, \ldots, n-1$. The operator $R$ has a regular singular point at zero and its coefficients $\widetilde{g}^{i}, i=0, \ldots, n-1$, are generalized analytic functions, i.e. they can be represented in the form $\widetilde{g}^{i}(s)=\int_{0}^{\infty} \psi^{i}(\alpha) s^{\alpha} e^{-\alpha / t} d \alpha$ with some entire functions $\psi^{i}, i=0, \ldots, n-1$, of exponential growth. Now, applying the Mellin transformation we obtain a convolution equation for the function $G(z):=\int_{0}^{t} w(s) s^{-z-1} d s$,

$$
Q(z) G(z)+\int_{0}^{\infty} A(\alpha, z) G(z-\alpha) e^{-\alpha / t} d \alpha=\Phi(z),
$$

where $A(\alpha, z)=\sum_{i=0}^{n-1} \psi^{i}(\alpha)(z-\alpha)^{i}$ and $\Phi$ is a polynomial determined by the Cauchy data. We solve the convolution equation by the method of successive approximations. Its solution $G$ is a holomorphic function on $\mathbb{C} \backslash \bigcup_{\mu=1}^{m}\left(\varrho_{\mu}+\overline{\mathbb{R}}_{+}\right)$, where $\varrho_{1}, \ldots, \varrho_{m}$ are the roots of $Q$. Furthermore, assuming that $\arg \left(\varrho_{\nu}-\varrho_{\mu}\right) \neq 0$ for any $1 \leq \nu<\mu \leq m$, the jump of $G$ across the half-line $\varrho_{\mu}+\overline{\mathbb{R}}_{+}$is a Laplace ultradistribution $S^{\mu}, \mu=1, \ldots, m$, on the half-line. Finally, the solution to the Cauchy problem for $R w=0$ is given by $w(s)=\sum_{\mu=1}^{m} S^{\mu}\left[s^{\cdot}\right]$ and putting $u(x)=w(s(x))$ we get the solution to 
the original Cauchy problem. A closer examination of the ultradistributions $S^{\mu}$ allows representing the solution $u$ in the form of Laplace integrals. This type of representation can be viewed as parallel to the one obtained by the multisummability method. The author believes that it can give a new insight into the Stokes phenomenon.

0. Notation. The open disc with centre at $z_{0} \in \mathbb{C}$ and radius $r>0$ is denoted by $B\left(z_{0} ; r\right)$ or simply by $B(r)$ if $z_{0}=0$.

By $\widetilde{B}(r)$ (resp. $\widetilde{\mathbb{C}}$ ) we denote the universal covering space of the punctured disc $B(r) \backslash\{0\}$ (resp. $\mathbb{C} \backslash\{0\}$ ). A point $z \in \widetilde{B}(r)$ is written as $z=|z| e^{i \arg z}$ with $0<|z|<r$ and $\arg z \in \mathbb{R}$.

For $\theta \in \mathbb{R}$ we set $l_{\theta}=\left(0, e^{i \infty \theta}\right)=\{z \in \mathbb{C} \backslash\{0\}: \arg z=\theta\}$. If $\theta=0$ then $l_{\theta}=\mathbb{R}_{+}$.

By a left (resp. right) tubular neighbourhood of a ray $\varrho+l_{\theta}, \varrho \in \mathbb{C}, \theta \in \mathbb{R}$, we mean a set $\left\{z: \operatorname{dist}\left(z, \varrho+l_{\theta}\right)<b, \theta<\arg (z-\varrho)<\theta+\pi / 2\right\}$ with some $b>0$ (resp. $\left.\left\{z: \operatorname{dist}\left(z, \varrho+l_{\theta}\right)<b, \theta-\pi / 2<\arg (z-\varrho)<\theta\right\}\right)$.

For $\varrho \in \mathbb{C}$ and $\theta^{-}<\theta^{+}$with $\theta^{+}-\theta^{-}<2 \pi$ we set

$$
S\left(\varrho ;\left(\theta^{-}, \theta^{+}\right)\right)=\left\{z \in \mathbb{C} \backslash\{\varrho\}: \theta^{-}<\arg (z-\varrho)<\theta^{+}\right\} .
$$

If $\theta^{+}-\theta^{-} \geq 2 \pi$ the set $S\left(\varrho ;\left(\theta^{-}, \theta^{+}\right)\right)$is interpreted as a subset of $\widetilde{C} \widetilde{\lceil\varrho\}}$.

For $\theta \in \mathbb{R}$ and $\omega \in \mathbb{R}$ we set

$$
\Omega_{\omega}^{\theta}=\{z \in \widetilde{\mathbb{C}}: \cos \theta \log |z|-\sin \theta \arg z<\omega\} .
$$

For $z \in \mathbb{C}$ we put $\langle z\rangle=1+|z|$.

1. Generalized analytic functions, the Laplace and Mellin transformations. To fit our purposes we slightly modify the theory of generalized analytic functions given in [Z], and the definitions of the Laplace and Mellin transformations. We do not give the proofs of the stated facts since the proofs follow the ones given in $[\mathrm{Z}]$, [Ł2] and [Ł3].

Fix $\varrho_{1}, \ldots, \varrho_{m} \in \mathbb{C}$ and $\theta \in \mathbb{R}$. Set $\Gamma_{\theta}=\bigcup_{\mu=1}^{m}\left(\varrho_{\mu}+\bar{l}_{\theta}\right)$. For $a \in \mathbb{R} e^{-i \theta}$ and $\omega \in \mathbb{R} e^{-i \theta}$ define

$$
\begin{array}{rlr}
L_{a}\left(\Gamma_{\theta}\right) & =\left\{\varphi \in C^{\infty}\left(\Gamma_{\theta}\right):\|\varphi\|_{a, h}=\sup _{0 \leq \alpha \leq h} \sup _{y \in \Gamma_{\theta}}\left|e^{-a y} D^{\alpha} \varphi(y)\right|<\infty\right. \\
L_{(\omega)}\left(\Gamma_{\theta}\right) & =\lim _{a<_{\theta} \omega} L_{a}\left(\Gamma_{\theta}\right), &
\end{array}
$$

where $a<_{\theta} \omega$ means that $a e^{i \theta}<\omega e^{i \theta}$. The dual space $L_{(\omega)}^{\prime}\left(\Gamma_{\theta}\right)$ of $L_{(\omega)}\left(\Gamma_{\theta}\right)$ is called the space of Laplace distributions on $\Gamma_{\theta}$. Replacing the norms $\|\varphi\|_{a, h}$ 
by

$$
\|\varphi\|_{a, h}^{\left(M_{p}\right)}=\sup _{\alpha \in \mathbb{N}_{0}} \sup _{y \in \Gamma_{\theta}} \frac{\left|e^{-a y} h^{\alpha} D^{\alpha} \varphi(y)\right|}{M_{\alpha}},
$$

where $\left(M_{p}\right)_{p=0}^{\infty}$ is a sequence of positive numbers satisfying conditions (M.1), (M.2) and (M.3) of $[\mathrm{K}]$, we obtain the space of Laplace ultradistributions $L_{(\omega)}^{\left(M_{p}\right) \prime}\left(\Gamma_{\theta}\right)$ (see also [€2]).

Observe that the function $\Gamma_{\theta} \ni y \mapsto \exp _{z}(y):=e^{y z}$ belongs to $L_{(\omega)}^{*}\left(\Gamma_{\theta}\right)$ where $*=\emptyset$ or $\left(M_{p}\right)$ if and only if $\operatorname{Re}\left(e^{i \theta} z\right)<\omega e^{i \theta}$. Thus, we can define the Laplace transform of $S \in L_{(\omega)}^{* \prime}\left(\Gamma_{\theta}\right)$ by

$$
\mathcal{L} S(z)=S\left[\exp _{z}\right] \quad \text { for } \operatorname{Re}\left(e^{i \theta} z\right)<\omega e^{i \theta} .
$$

Note that $\mathcal{L} S(-1 / x)$ is defined in the $\operatorname{disc}\left(2 \omega e^{i \theta}\right)^{-1} B\left(e^{i \theta} ; 1\right)$ if $\omega e^{i \theta}<0$, in the half-plane $\operatorname{Re}\left(x e^{-i \theta}\right)>0$ if $\omega=0$, and outside $\left(2 \omega e^{i \theta}\right)^{-1} B\left(-e^{i \theta} ; 1\right)$ if $0<\omega e^{i \theta}$.

Analogously, the function $\Gamma_{\theta} \ni y \mapsto \varphi_{s}(y):=s^{y}$ belongs to $L_{(\omega)}^{*}\left(\Gamma_{\theta}\right)$ iff $s \in \Omega_{\omega e^{i \theta}}^{\theta}:=\left\{s \in \widetilde{\mathbb{C}}: \cos \theta \log |s|-\sin \theta \arg s<\omega e^{i \theta}\right\}$. So, we can define the Taylor transform of $S \in L_{(\omega)}^{* \prime}\left(\Gamma_{\theta}\right)$ by

$$
\mathcal{T} S(s)=S\left[\varphi_{s}\right] \quad \text { for } s \in \Omega_{\omega e^{i \theta}}^{\theta} .
$$

We call the image of $L_{(\omega)}^{* \prime}\left(\Gamma_{\theta}\right)$ under the Taylor transformation the space of generalized analytic functions determined by $L_{(\omega)}^{* \prime}\left(\Gamma_{\theta}\right)$ and denote it by $\operatorname{GAF}\left(L_{(\omega)}^{* \prime}\left(\Gamma_{\theta}\right)\right)$. If $\left(\varrho_{\nu}+l_{\theta}\right) \cap\left(\varrho_{\mu}+l_{\theta}\right)=\emptyset$ for $1 \leq \nu<\mu \leq m$, we have a natural decomposition

$$
\operatorname{GAF}\left(L_{(\omega)}^{* \prime}\left(\Gamma_{\theta}\right)\right)=\bigoplus_{\mu=1}^{m} \operatorname{GAF}\left(L_{(\omega)}^{* \prime}\left(\varrho_{\mu}+\bar{l}_{\theta}\right)\right)=\bigoplus_{\mu=1}^{m} s^{\varrho_{\mu}} \cdot \operatorname{GAF}\left(L_{(\omega)}^{* \prime}\left(\bar{l}_{\theta}\right)\right) .
$$

The space $\operatorname{GAF}\left(L_{(\omega)}^{* \prime}\left(\bar{l}_{\theta}\right)\right)$ can be characterized (cf. [€2], Th. 6) as the set of $w \in \mathcal{O}\left(\Omega_{\omega e^{i \theta}}^{\theta}\right)$ such that for any $a<_{\theta} \omega$ one can find $k<\infty$ such that

$$
|w(s)| \leq \begin{cases}C(1+|\log s|)^{k} & \text { for } s \in \bar{\Omega}^{\theta} e^{i \theta} \text { if } *=\emptyset, \\ C \exp \{M(k|\log s|)\} & \text { for } s \in \bar{\Omega}_{a e^{i \theta}}^{\theta} \text { if } *=\left(M_{p}\right),\end{cases}
$$

where $M$ is the associated function of the sequence $\left(M_{p}\right)$ defined by

$$
M(\varrho)=\sup _{p \in \mathbb{N}_{0}} \log \frac{\varrho^{p} M_{0}}{M_{p}} \quad \text { for } \varrho>0 .
$$

Fix $t \in \Omega_{\omega e^{i \theta}}^{\theta}$ and $\varrho \in \mathbb{C}$. We define the Mellin transform of $w \in$ $\operatorname{GAF}\left(L_{(\omega)}^{* \prime}\left(\varrho+\bar{l}_{\theta}\right)\right)$ by

$$
\mathcal{M}_{t}^{\theta} w(z)=\int_{\gamma_{t}^{\theta}} w(s) s^{-z-1} d s
$$


where $\gamma_{t}^{\theta}=\left\{s \in \widetilde{\mathbb{C}}: s=t \exp \left\{-e^{-i \theta} r\right\}, 0 \leq r<\infty\right\}$ with the orientation reverse to that induced by the above parametrization. Then $\mathcal{M}_{t}^{\theta} w$ is holomorphic on $\left\{\operatorname{Re}\left((z-\varrho) e^{-i \theta}\right)<0\right\}$. Since the integration curve $\gamma_{t}^{\theta}$ in (4) can be replaced by $\gamma_{t}^{\theta^{\prime}}$ for any $\left|\theta-\theta^{\prime}\right| \leq \pi / 2$ we conclude that $\mathcal{M}_{t}^{\theta} w \in \mathcal{O}\left(\mathbb{C} \backslash\left(\varrho+\bar{l}_{\theta}\right)\right)$. Furthermore (cf. [Ł3], Th. 4, [Z], Th. 10.1), there exists $C<\infty$ such that for $0<\operatorname{dist}\left(z, \varrho+\bar{l}_{\theta}\right) \leq 1$,

$$
\left|\mathcal{M}_{t}^{\theta} w(z)\right| \leq \begin{cases}C\left|t^{\varrho-z}\right|\left(\operatorname{dist}\left(z, \varrho+\bar{l}_{\theta}\right)\right)^{-C} & \text { if } *=\emptyset, \\ C\left|t^{\varrho-z}\right| \exp \left\{M^{*}\left(\frac{C}{\operatorname{dist}\left(z, \varrho+\bar{l}_{\theta}\right)}\right)\right\} & \text { if } *=\left(M_{p}\right),\end{cases}
$$

where $M^{*}$ is the growth function of the sequence $\left(M_{p}\right)$ given by

$$
M^{*}(\varrho)=\sup _{p \in \mathbb{N}_{0}} \log \frac{\varrho^{p} p ! M_{0}}{M_{p}} \quad \text { for } \varrho>0 .
$$

Moreover, the boundary value of $\mathcal{M}_{t}^{\theta} w, S=b\left(\mathcal{M}_{t}^{\theta} w\right)$, belongs to $L_{(\omega)}^{* \prime}\left(\varrho+\bar{l}_{\theta}\right)$ and $w=(2 \pi i)^{-1} \mathcal{T} S$.

Conversely (cf. [Ł3], Th. 5), if $G \in \mathcal{O}\left(\mathbb{C} \backslash\left(\varrho+\bar{l}_{\theta}\right)\right)$ satisfies (5) (with $G$ in place of $\left.\mathcal{M}_{t}^{\theta} w\right)$ for $0<\operatorname{dist}\left(z, \varrho+\bar{l}_{\theta}\right) \leq 1$ and $|G(z)| \leq C\left|t^{\varrho-z}\right| /\langle z\rangle$ for $\operatorname{dist}\left(z, \varrho+\bar{l}_{\theta}\right) \geq 1$ then $G=\mathcal{M}_{t}^{\theta} w$ with a unique $w$ given by $w=$ $(2 \pi i)^{-1} \mathcal{T} b(G)$.

Analogously, using the decomposition (3), we define the Mellin transform of $w \in \operatorname{GAF}\left(L_{(\omega)}^{* \prime}\left(\Gamma_{\theta}\right)\right)$, which is a holomorphic function on $\mathbb{C} \backslash \Gamma_{\theta}$ and satisfies appropriate estimates.

The Mellin transformation has the following operational property, which makes it useful in the study of the Cauchy problem.

If $w \in \operatorname{GAF}\left(L_{(\omega)}^{* \prime}\left(\Gamma_{\theta}\right)\right)$ and $t \in \Omega_{\omega e^{i \theta}}^{\theta}$ then for $i \in \mathbb{N}_{0}$,

$$
\mathcal{M}_{t}^{\theta}\left(\left(s \frac{d}{d s}\right)^{i} w\right)(z)=z^{i} \mathcal{M}_{t}^{\theta} w(z)+W_{i}(z) \quad \text { for } z \in \mathbb{C} \backslash \Gamma_{\theta},
$$

where $W_{i}$ is a polynomial of degree $\leq i-1$ depending on $w(t), \ldots, w^{(i-1)}(t)$.

2. The main result. Let $P$ be a differential operator (1) with coefficients analytic in $B(r), r>0$. Assume that $P$ is 1-regular and $a^{n}(x)=x^{2 n}$. Then for $i=0, \ldots, n-1, a^{i}(x)=\sum_{j=2 i}^{\infty} a_{j}^{i} x^{j}$ for $|x|<r$. Furthermore, it follows by Lemma 1.3 of Chapter 4 of $[\mathrm{T}]$ that $P$ can be written in the form (2), where $Q(z)=z^{n}+\sum_{i=0}^{n-1} a_{2 i}^{i} z^{i}$ and $g^{i}(x)=\sum_{j=1}^{\infty} g_{j}^{i} x^{j}$ for $|x|<r, i=0, \ldots, n-1$. Fix $0<t<r$ and consider the Cauchy problem

$$
\left\{\begin{array}{l}
P u=0, \\
u(t)=u_{0}, \ldots, u^{(n-1)}(t)=u_{n-1} .
\end{array}\right.
$$


It is well known that the solution $u$ of $(8)$ is unique and it extends holomorphically to a function on $\widetilde{B}(r)$. Our aim is to represent the $u$ in the form of Laplace type integrals. To formulate the main result denote by $\varrho_{1}, \ldots, \varrho_{m}$ the roots of $Q$ with multiplicities $k_{1}, \ldots, k_{m}$, respectively. Define the set $\Theta_{\mathrm{s}}$ of singular directions by

$$
\Theta_{\mathrm{s}}=\left\{\theta \in \mathbb{R}: \theta \bmod (2 \pi)=\arg \left(\varrho_{\nu}-\varrho_{\mu}\right) \text { for some } 1 \leq \nu \neq \mu \leq m\right\}
$$

$\left(\Theta_{\mathrm{s}}=\emptyset\right.$ if $\left.m=1\right)$. Choose $\theta \notin \Theta_{\mathrm{s}}$ such that $t \in(r / 2) B\left(e^{i \theta} ; 1\right)$ and denote by $\theta^{-}$(resp. $\theta^{+}$) the greatest (resp. smallest) singular direction less (resp. greater) than $\theta\left(\theta^{ \pm}= \pm \infty\right.$ if $\left.\Theta_{\mathrm{s}}=\emptyset\right)$.

Main Theorem. Let $P$ be a 1-regular operator (2). Fix $0<t<r$ and retain the preceding notations. Then the unique solution $u$ of the Cauchy problem (8) is given by

$$
u(x)=\sum_{\mu=1}^{m} \mathcal{L} S^{\mu}(1 / t-1 / x) \quad \text { for } x \in(r / 2) B\left(e^{i \theta} ; 1\right),
$$

with a unique $S^{\mu} \in L_{(\omega)}^{* \prime}\left(\varrho_{\mu}+\bar{l}_{\theta}\right)(\mu=1, \ldots, m)$, where $\omega=(\cos (\theta / t)-$ $1 / r) e^{-i \theta}$ and

$$
*= \begin{cases}\emptyset & \text { if } k_{\mu}=1 \\ p !(p / \log p)^{p /\left(k_{\mu}-1\right)} & \text { if } k_{\mu}>1 .\end{cases}
$$

Furthermore, $S^{\mu}, \mu=1, \ldots, m$, restricted to $\varrho_{\mu}+l_{\theta}$ extends holomorphically to a function $\Psi^{\mu} \in \mathcal{O}\left(S\left(\varrho_{\mu} ;\left(\theta^{-}, \theta^{+}\right)\right)\right)$such that for any $r^{\prime}<r$ and $\theta^{-}<\widetilde{\theta}^{-}$ $<\widetilde{\theta}^{+}<\theta^{+}$,

$$
\left|\Psi^{\mu}\left(\varrho_{\mu}+\gamma\right) e^{\gamma / t}\right| \leq \begin{cases}C|\gamma|^{-C} \exp \left\{|\gamma| / r^{\prime}\right\} & \text { if } k_{\mu}=1 \\ C \exp \left\{\frac{C}{|\gamma|^{k_{\mu}-1}} \log \frac{C}{|\gamma|}+\frac{|\gamma|}{r^{\prime}}\right\} & \text { if } k_{\mu}>1\end{cases}
$$

for $\widetilde{\theta}^{-} \leq \arg \gamma \leq \widetilde{\theta}^{+}$with some $C<\infty$.

Thus, for any $\theta^{-}<\theta^{\prime}<\theta^{+}, u$ can be written in the form

$$
\begin{aligned}
& u(x)=\sum_{\mu=1}^{m} e^{-\varrho_{\mu}(1 / t-1 / x)} \operatorname{reg} \int_{l_{\theta^{\prime}}} \Psi^{\mu}\left(\varrho_{\mu}+\gamma\right) e^{\gamma / t-\gamma / x} d \gamma \\
& \text { for } x \in(r / 2) B\left(e^{i \theta^{\prime}} ; 1\right),
\end{aligned}
$$

where the regularization of the integral is distributional if $k_{\mu}=1$ and ultradistributional of class $p !(p / \log p)^{p /\left(k_{\mu}-1\right)}$ if $k_{\mu}>1$.

REMARK. We conjecture that $\Psi^{\mu}$ is a multivalued holomorphic function on $\mathbb{C}$ with the set of branching points $\left\{\varrho_{1}, \ldots, \varrho_{\mu}\right\}$. 
3. Auxiliary lemmas. In the proof of the main theorem we shall use the following lemmas.

Lemma 1. For $\nu \in \mathbb{N}$ put

$$
I^{\nu}(\gamma, z)=\int_{T^{\nu}(\gamma)} \frac{d \alpha}{\left\langle z-\alpha_{1}\right\rangle \ldots\left\langle z-\alpha_{1}-\ldots-\alpha_{\nu}\right\rangle} \quad \text { for } \gamma \in \mathbb{R}_{+}, z \in \mathbb{C}
$$

with $T^{\nu}(\gamma)=\left\{\alpha \in\left(\mathbb{R}_{+}\right)^{\nu}: \alpha_{1}+\ldots+\alpha_{\nu} \leq \gamma\right\}$. Then

$$
\left|I^{\nu}(\gamma, z)\right| \leq \frac{2^{\nu}}{\nu !} \log ^{\nu}(1+|\gamma|) \quad \text { for } \gamma \in \mathbb{R}_{+}, z \in \mathbb{C} .
$$

Proof. We can consider only the case $z=x \in \mathbb{R}_{+}$. Let $0<\gamma \leq x$. By induction we show that

$$
I^{\nu}(\gamma, x)=\frac{1}{\nu !} \log ^{\nu}\left(\frac{1+x}{1+x-\gamma}\right)
$$

which is bounded by $\frac{1}{\nu !} \log ^{\nu}(1+\gamma)$. In fact, $I^{1}(\gamma, x)=\log \frac{1+x}{1+x-\gamma}$ and for $\nu \geq 2$ we derive

$$
\begin{aligned}
I^{\nu}(\gamma, x) & =\int_{0}^{\gamma} \frac{1}{1+x-\alpha_{1}} I^{\nu-1}\left(\gamma-\alpha_{1}, x-\alpha_{1}\right) d \alpha_{1} \\
& =\frac{1}{(\nu-1) !} \int_{0}^{\gamma} \frac{\log ^{\nu-1}\left(\frac{1+x-\alpha_{1}}{1+x-\gamma}\right)}{1+x-\alpha_{1}} d \alpha_{1}=\frac{1}{\nu !} \log ^{\nu}\left(\frac{1+x}{1+x-\gamma}\right) .
\end{aligned}
$$

Now let $0<x \leq \gamma$. We observe that $T^{\nu}(\gamma)=\bigcup_{k=0}^{\nu} T_{k}^{\nu}(\gamma)$ with $T_{k}^{\nu}(\gamma)=$ $\left\{\alpha \in \mathbb{R}_{+}^{\nu}: \alpha_{1} \leq x, \ldots, \alpha_{1}+\ldots+\alpha_{\nu-k} \leq x, x \leq \alpha_{1}+\ldots+\alpha_{\nu-k+1}, \alpha_{1}+\right.$ $\left.\ldots+\alpha_{\nu} \leq \gamma\right\}$. Now for $k \in\{0,1, \ldots, \nu\}$ we compute

$$
\begin{aligned}
\int_{T_{k}^{\nu}(\gamma)} \frac{1}{1+} & x-\alpha_{1} \\
& \quad \times \frac{1}{1+\alpha_{1}+\ldots+\alpha_{\nu-k+1}-x} \cdots \frac{1}{1+\alpha_{1}+\ldots+\alpha_{\nu}-x} d \alpha \\
= & \int_{T^{\nu-k}(x)} \frac{1}{1+x-\alpha_{1}} \cdots \frac{1}{1+x-\alpha_{1}-\ldots-\alpha_{\nu-k}} d \alpha \\
& \quad \times \quad \int_{T^{k}(\gamma-x)} \frac{1}{1+\beta_{1}} \cdots \frac{1}{1+\beta_{1}+\ldots+\beta_{k}} d \beta \\
= & \frac{1}{(\nu-k) !} \log ^{\nu-k}(1+x) \cdot \frac{1}{k !} \log ^{k}(1+\gamma-x) .
\end{aligned}
$$

So 


$$
\begin{aligned}
I^{\nu}(\gamma, x) & =\sum_{k=0}^{\nu} \frac{1}{(\nu-k) ! k !} \log ^{\nu-k}(1+x) \cdot \log ^{k}(1+\gamma-x) \\
& =\frac{1}{\nu !}(\log (1+x)+\log (1+\gamma-x))^{\nu},
\end{aligned}
$$

which is bounded by $\frac{2^{\nu}}{\nu !} \log ^{\nu}(1+\gamma)$.

Lemma 2. Let $|\Psi(\gamma)| \leq C e^{|\gamma| / r}$ for $\gamma \in e^{i \theta}+l_{\theta}$ with $r>0$. Then the integral

$$
w_{\theta}(s)=\int_{e^{i \theta}+l_{\theta}} \Psi(\gamma) s^{\gamma} e^{-\gamma} d \gamma
$$

converges on the set of $s \in \widetilde{\mathbb{C}}$ such that $s / e \in \Omega_{-1 / r}^{\theta}$ and $u_{\theta}(x)=$ $w_{\theta}(\exp \{1-1 / x\})$ is defined in the disc $(r / 2) B\left(e^{i \theta} ; 1\right)$.

P r o of. Indeed

$$
w_{\theta}(s)=\int_{1}^{\infty} \Psi\left(t e^{i \theta}\right)(s / e)^{t e^{i \theta}} e^{i \theta} d t
$$

and the integral converges if

$$
1 / r+\operatorname{Re}\left(e^{i \theta} \log (s / e)\right)=1 / r+\cos \theta \log (|s| / e)-\sin \theta \arg s<0 .
$$

To prove the second statement observe that for $s=\exp \{1-1 / x\}$ we have $\log (|s| / e)=-\operatorname{Re}(1 / x)=-\operatorname{Re} x /|x|^{2}$ and $\arg s=-\operatorname{Im}(1 / x)=$ $\operatorname{Im} x /|x|^{2}$. So, if $s / e \in \Omega_{-1 / r}^{\theta}$ then $x$ satisfies $(\cos \theta \operatorname{Re} x+\sin \theta \operatorname{Im} x)|x|^{-2}=$ $\operatorname{Re}\left(e^{-i \theta} x\right)|x|^{-2}>r^{-1}$ and hence $x \in(r / 2) B\left(e^{i \theta} ; 1\right)$.

Lemma 3. Let $s>0, M_{0}=M_{1}=1$ and $M_{p}=p !(p / \log p)^{p s}$ for $p \in \mathbb{N}$, $p \geq 2$. Then $M^{*}(\varrho) \sim \varrho^{1 / s} \log \varrho$ as $\varrho \rightarrow \infty$.

Pro of. By (8) we have, for $\varrho>1$,

$$
M^{*}(\varrho)=\max \left(\log \varrho, \sup _{p \in \mathbb{N}, p \geq 2} p(\log \varrho+s \log \log p-s \log p)\right) .
$$

To compute the supremum define

$$
g(\varrho, x)=x(\log \varrho+s \log \log x-s \log x) \quad \text { for } x>1, \varrho>0 .
$$

Since $g_{x}^{\prime}(\varrho, x)=\log \varrho+s \log \log x-s \log x+s / \log x-s$, for $\varrho \geq e^{s}$ there exists a unique $x(\varrho) \geq e$ such that $g_{x}^{\prime}(\varrho, x(\varrho))=0$. Put $g(\varrho)=g(\varrho, x(\varrho))$ for $\varrho \geq e^{s}$. Then $g(\varrho)=s x(\varrho)(1-1 / \log x(\varrho))$ and so $g(\varrho) \sim x(\varrho)$ as $\varrho \rightarrow \infty$. Put

$$
f(x)=\left(\frac{e x}{\log x} \exp \left\{-\frac{1}{\log x}\right\}\right)^{s} \text { and } h(\varrho)=\varrho^{1 / s} \log \varrho^{1 / s} .
$$

Then for $\varrho>e^{s}, e^{-s} f(h(\varrho)) \leq \varrho \leq 2^{s-1} f(h(2 \varrho))$. Since $x(\varrho)=f^{-1}(\varrho)$ this implies $h(\varrho) \sim x(\varrho)$ as $\varrho \rightarrow \infty$. Finally, in a standard way (see [Ł1]) we show that $M^{*}(\varrho) \sim g(\varrho)$ as $\varrho \rightarrow \infty$. 
4. Proof of the main theorem. Let $P$ be given by (2), where $Q$ is a polynomial of degree $n$ and $g^{i}(x)=\sum_{j=1}^{\infty} g_{j}^{i} x^{j}, i=0, \ldots, n-1$, are functions analytic in the disc $B(r), r>0$. Consider the Cauchy problem (8). Putting, if necessary, $x^{\prime}=x / t$ we can assume that $t=1$ and $r>1$. Observe that by the change of independent variable $s(x)=\exp \{1-1 / x\},(8)$ is transformed into

$$
\left\{\begin{array}{l}
Q\left(s \frac{d}{d s}\right) w+\sum_{i=0}^{n-1} \widetilde{g}^{i}(s)\left(s \frac{d}{d s}\right)^{i} w=0, \\
w(1)=w_{0}, \ldots, w^{(n-1)}(1)=w_{n-1},
\end{array}\right.
$$

where $\widetilde{g}^{i}(s)=g^{i}\left((-\log (s / e))^{-1}\right), i=0, \ldots, n-1, w(s)=u\left((-\log (s / e))^{-1}\right)$, $w_{0}=u_{0}, w_{1}=u_{1}, w_{2}=u_{1}+u_{2}$ and so on.

Since $\lim _{s \rightarrow 0} \widetilde{g}^{i}(s)=0$ for $i=0, \ldots, n-1$, we have obtained an equation with a regular singular point at zero, but with coefficients which are generalized analytic functions of the form (cf. [Z], Th. 14.1)

$$
\widetilde{g}^{i}(s)=\int_{l_{\theta}} \psi^{i}(\alpha) s^{\alpha} e^{-\alpha} d \alpha \quad \text { for } s / e \in \Omega_{-1 / r}^{\theta}, \theta \in \mathbb{R},
$$

where $\psi^{i}(\alpha)=\sum_{j=1}^{\infty} g_{j}^{i} \alpha^{j-1} /(j-1)$ ! for $\alpha \in \mathbb{C}$ is the Borel transform of $g^{i}$, which is an entire function satisfying $\left|\psi^{i}(\alpha)\right| \leq C_{r^{\prime}} \exp \left\{|\alpha| / r^{\prime}\right\}$ for any $r^{\prime}<r(i=0, \ldots, n-1)$.

The equations of this type were studied by Bogdan Ziemian in [Z]. Under suitable conditions he proved the existence of generalized analytic solutions with positive radii of convergence. However his theorem ([Z], Theorem 16.2) cannot be applied here without additional assumptions on the functions $g^{i}$ and does not guarantee that the radius of convergence of a solution is greater than 1.

We shall solve (13) by applying the Mellin transformation. Fix a nonsingular direction $\theta \notin \Theta_{\mathrm{s}}$ such that $\cos \theta>1 / r$ (this assumption ensures that $\left.1 \in \Omega_{-1 / r}^{\theta}\right)$. Observe that by (14) and (7),

$$
\begin{aligned}
\mathcal{M}_{1}^{\theta}\left(\widetilde{g}^{i}(s)\left(s \frac{d}{d s}\right)^{i} w\right)(z) & \\
& =\int_{l_{\theta}} \psi^{i}(\alpha)\left((z-\alpha)^{i} \mathcal{M}_{1}^{\theta} w(z-\alpha)+W_{i}(z-\alpha)\right) e^{-\alpha} d \alpha \\
& =\int_{l_{\theta}} \psi^{i}(\alpha)(z-\alpha)^{i} \mathcal{M}_{1}^{\theta} w(z-\alpha) e^{-\alpha} d \alpha+\widetilde{W}_{i}(z),
\end{aligned}
$$

where $\widetilde{W}_{i}$ is a polynomial of degree $\leq i-1, i=0, \ldots, n-1$. Thus applying the Mellin transformation to (13) we get the convolution equation 


$$
Q(z) G_{\theta}(z)+\int_{l_{\theta}} A^{0}(\alpha, z) G_{\theta}(z-\alpha) e^{-\alpha} d \alpha=\Phi(z)
$$

where

$$
G_{\theta}(z)=\mathcal{M}_{1}^{\theta} w(z), \quad A^{0}(\alpha, z)=\sum_{i=0}^{n-1} \psi^{i}(\alpha)(z-\alpha)^{i}
$$

and $\Phi$ is a polynomial of degree $\leq n-1$ depending on $w_{0}, \ldots, w_{n-1}$.

We solve (15) by the approximation scheme

$$
\begin{aligned}
G_{\theta}^{0}(z) & =\frac{\Phi(z)}{Q(z)}, \\
G_{\theta}^{\nu+1}(z) & =\frac{1}{Q(z)}\left\{\Phi(z)-\int_{l_{\theta}} A^{0}(\alpha, z) G_{\theta}^{\nu}(z-\alpha) e^{-\alpha} d \alpha\right\}, \quad \nu \in \mathbb{N} .
\end{aligned}
$$

Put $\widetilde{G}_{\theta}^{\nu+1}=G_{\theta}^{\nu+1}-G_{\theta}^{\nu}$ for $\nu \in \mathbb{N}_{0}$. Then we find

$$
\widetilde{G}_{\theta}^{\nu+1}(z)=\frac{(-1)^{\nu+1}}{Q(z)} \int_{l_{\theta}} A_{\theta}^{\nu}(\gamma, z) \frac{\Phi(z-\gamma)}{Q(z-\gamma)} e^{-\gamma} d \gamma
$$

where for $\gamma \in l_{\theta}, \nu \in \mathbb{N}$,

$$
\begin{aligned}
A_{\theta}^{\nu}(\gamma, z)= & \int_{\substack{\alpha_{1} \in l_{\theta} \\
\left|\alpha_{1}\right| \leq|\gamma|}} \frac{A^{0}\left(\alpha_{1}, z\right) A^{\nu-1}\left(\gamma-\alpha_{1}, z-\alpha_{1}\right)}{Q\left(z-\alpha_{1}\right)} d \alpha_{1} \\
= & \int_{T_{\theta}^{\nu}(\gamma)} \frac{A^{0}\left(\alpha_{1}, z\right)}{Q\left(z-\alpha_{1}\right)} \ldots \frac{A^{0}\left(\alpha_{\nu}, z-\alpha_{1}-\ldots-\alpha_{\nu-1}\right)}{Q\left(z-\alpha_{1}-\ldots-\alpha_{\nu}\right)} \\
& \times A^{0}\left(\gamma-\alpha_{1}-\ldots-\alpha_{\nu}, z-\alpha_{1}-\ldots-\alpha_{\nu}\right) d \alpha
\end{aligned}
$$

with $T_{\theta}^{\nu}(\gamma)=\left\{\alpha \in\left(l_{\theta}\right)^{\nu}:\left|\alpha_{1}+\ldots+\alpha_{\nu}\right| \leq|\gamma|\right\}, \gamma \in l_{\theta}$.

Assume that $\operatorname{dist}\left(z, \bigcup_{\mu=1}^{m}\left(\varrho_{\mu}+l_{\theta}\right)\right) \geq b$ with some $b>0$. Then we can find $C_{b}$ such that $\langle z\rangle^{n} \leq C_{b}|Q(z)|$. Since $\left|A^{0}(\alpha, z)\right| \leq C e^{|\alpha| / r^{\prime}}\langle z\rangle^{n-1}$ we derive

$$
\begin{gathered}
\frac{\left|A^{0}\left(\alpha_{1}, z\right)\right|}{|Q(z)|} \leq \frac{C C_{b} e^{\left|\alpha_{1}\right| / r^{\prime}}}{\langle z\rangle}, \quad \frac{\left|A^{0}\left(\alpha_{2}, z-\alpha_{1}\right)\right|}{\left|Q\left(z-\alpha_{1}\right)\right|} \leq \frac{C C_{b} e^{\left|\alpha_{2}\right| / r^{\prime}}}{\left\langle z-\alpha_{1}\right\rangle}, \ldots, \\
\frac{\left|A^{0}\left(\alpha_{\nu}, z-\alpha_{1}-\ldots-\alpha_{\nu-1}\right)\right|}{\left|Q\left(z-\alpha_{1}-\ldots-\alpha_{\nu-1}\right)\right|} \leq \frac{C C_{b} e^{\left|\alpha_{\nu}\right| / r^{\prime}}}{\left\langle z-\alpha_{1}-\ldots-\alpha_{\nu-1}\right\rangle}, \\
\frac{\left|A^{0}\left(\gamma-\alpha_{1}-\ldots-\alpha_{\nu}, z-\alpha_{1}-\ldots-\alpha_{\nu}\right)\right|}{\left|Q\left(z-\alpha_{1}-\ldots-\alpha_{\nu}\right)\right|} \leq \frac{C C_{b} e^{\left|\gamma-\alpha_{1}-\ldots-\alpha_{\nu}\right| / r^{\prime}}}{\left\langle z-\alpha_{1}-\ldots-\alpha_{\nu}\right\rangle} .
\end{gathered}
$$

So by Lemma 1 ,

$$
\frac{\left|A_{\theta}^{\nu}(\gamma, z)\right|}{|Q(z)|} \leq \frac{\left(C C_{b}\right)^{\nu+1}}{\langle z\rangle} e^{|\gamma| / r^{\prime}} \frac{2^{\nu}}{\nu !} \log ^{\nu}(1+|\gamma|) .
$$


Thus

$$
G_{\theta}(z)=\frac{\Phi(z)}{Q(z)}+\int_{l_{\theta}} \frac{A_{\theta}(\gamma, z)}{Q(z)} \cdot \frac{\Phi(z-\gamma)}{Q(z-\gamma)} e^{-\gamma} d \gamma
$$

where $A_{\theta}(\gamma, z)=\sum_{\nu=0}^{\infty}(-1)^{\nu+1} A_{\theta}^{\nu}(\gamma, z)$ satisfies, with $K=2 C C_{b}$,

$$
\begin{aligned}
\frac{\left|A_{\theta}(\gamma, z)\right|}{|Q(z)|} \leq & \frac{C C_{b}}{\langle z\rangle} e^{|\gamma| / r^{\prime}}(1+|\gamma|)^{K} \\
& \quad \text { for } \gamma \in l_{\theta}, \operatorname{dist}\left(z, \bigcup_{\mu=1}^{m}\left(\varrho_{\mu}+l_{\theta}\right)\right) \geq b .
\end{aligned}
$$

Finally, since $|\Phi(z)|\langle z\rangle \leq C_{b}|Q(z)|$ for $\operatorname{dist}\left(z,\left\{\varrho_{1}, \ldots, \varrho_{m}\right\}\right) \geq b$ we get, with some $C<\infty$,

$$
\left|G_{\theta}(z)\right| \leq \frac{C}{\langle z\rangle} \quad \text { for } \operatorname{dist}\left(z, \bigcup_{\mu=1}^{m}\left(\varrho_{\mu}+\bar{l}_{\theta}\right)\right) \geq b .
$$

Now assume that $z$ is close to $\varrho_{\mu}$ with a fixed $\mu \in\{1, \ldots, m\}$. To shorten notation put $k=k_{\mu}$. Assume $d \leq\left|z-\varrho_{\mu}\right| \leq b,\left|\arg \left(z-\varrho_{\mu}-\theta\right)\right| \geq \beta$ with some $\beta>0$ and $0<d<b \leq 1$ with $\operatorname{dist}\left(\varrho_{\mu}+\bar{l}_{\theta}, \bigcup_{\nu \neq \mu}\left(\varrho_{\nu}+\bar{l}_{\theta}\right)\right) \geq 2 b$. Since for $\alpha \in l_{\theta}$ we have $\langle z-\alpha\rangle^{n-k}\left|z-\varrho_{\mu}-\alpha\right|^{k} \leq C|Q(z-\alpha)|,\langle z-\alpha\rangle^{k-1} \leq C_{1}\langle\alpha\rangle^{k-1}$ and $(d+|\alpha|)^{k} \leq C_{2}\left|z-\varrho_{\mu}-\alpha\right|^{k}$ we get, with a constant $C$ independent of $d$,

$$
\begin{gathered}
\frac{\left|A^{0}\left(\alpha_{1}, z\right)\right|}{|Q(z)|} \leq \frac{C e^{\left|\alpha_{1}\right| / r^{\prime}}}{d^{k}}, \\
\frac{\left|A^{0}\left(\alpha_{2}, z-\alpha_{1}\right)\right|}{\left|Q\left(z-\alpha_{1}\right)\right|} \leq C e^{\left|\alpha_{2}\right| / r^{\prime}} \frac{\left\langle z-\alpha_{1}\right\rangle^{k-1}}{\left|z-\varrho_{\mu}-\alpha_{1}\right|^{k}} \leq C e^{\left|\alpha_{2}\right| / r^{\prime}} \frac{C_{1} C_{2}\left\langle\alpha_{1}\right\rangle^{k-1}}{\left(d+\left|\alpha_{1}\right|\right)^{k}}, \ldots, \\
\frac{\left|A^{0}\left(\alpha_{\nu}, z-\alpha_{1}-\ldots-\alpha_{\nu-1}\right)\right|}{\left|Q\left(z-\alpha_{1}-\ldots-\alpha_{\nu-1}\right)\right|} \leq C e^{\left|\alpha_{\nu}\right| / r^{\prime}} \frac{C_{1} C_{2}\left\langle\alpha_{1}+\ldots+\alpha_{\nu-1}\right\rangle^{k-1}}{\left(d+\left|\alpha_{1}+\ldots+\alpha_{\nu-1}\right|\right)^{k}}, \\
\frac{\left|A^{0}\left(\gamma-\alpha_{1}-\ldots-\alpha_{\nu}, z-\alpha_{1}-\ldots-\alpha_{\nu}\right)\right|}{\left|Q\left(z-\alpha_{1}-\ldots-\alpha_{\nu}\right)\right|} \\
\leq C e^{\left|\gamma-\alpha_{1}-\ldots-\alpha_{\nu}\right| / r^{\prime}} \frac{C_{1} C_{2}\left\langle\alpha_{1}+\ldots+\alpha_{\nu}\right\rangle^{k-1}}{\left(d+\left|\alpha_{1}+\ldots+\alpha_{\nu}\right|\right)^{k}} .
\end{gathered}
$$

So for $\gamma \in l_{\theta}$,

$$
\begin{aligned}
\frac{\left|A_{\theta}^{\nu}(\gamma, z)\right|}{|Q(z)|} \leq & \frac{C}{d^{k}}\left(C C_{1} C_{2}\right)^{\nu} e^{|\gamma| / r^{\prime}} \\
& \times \int_{T_{\theta}^{\nu}(\gamma)} \frac{\left\langle\alpha_{1}\right\rangle^{k-1} \ldots\left\langle\alpha_{1}+\ldots+\alpha_{\nu}\right\rangle^{k-1}}{\left(d+\left|\alpha_{1}\right|\right)^{k} \ldots\left(d+\left|\alpha_{1}+\ldots+\alpha_{\nu}\right|\right)^{k}} d \alpha \\
\leq & \frac{C}{d^{k}} e^{|\gamma| / r^{\prime}} \frac{1}{\nu !}\left(\frac{L}{d^{k-1}} \log \frac{d+|\gamma|}{d}\right)^{\nu},
\end{aligned}
$$


where $L=C C_{1} C_{2}$ (for $k>1$ we use $\left.\langle\alpha\rangle^{k-1} d^{k-1} \leq(d+|\alpha|)^{k-1}\right)$. Thus

$$
\frac{\left|A_{\theta}(\gamma, z)\right|}{|Q(z)|} \leq \frac{C}{d^{k}} e^{|\gamma| / r^{\prime}}\left(\frac{d+|\gamma|}{d}\right)^{L / d^{k-1}} .
$$

Finally, since

$$
\int_{l_{\theta}}\left(1+\frac{|\gamma|}{d}\right)^{L / d^{k-1}} e^{|\gamma| / r^{\prime}} e^{-|\gamma|}|d \gamma| \leq C\left(\frac{C}{d}\right)^{L / d^{k-1}} \Gamma\left(\frac{L}{d^{k-1}}+1\right)
$$

(here $C=2 r^{\prime} /\left(r^{\prime}-1\right)$ and $\Gamma$ is the Euler function), we obtain, with $C$ independent of $d$,

$$
\left|G_{\theta}(z)\right| \leq \begin{cases}C d^{-L-1} & \text { if } k=1, \\ \frac{C}{d^{k}} \exp \left\{\frac{L}{d^{k-1}} \log \frac{C L}{d^{k}}\right\} & \text { if } k>1\end{cases}
$$

for $d \leq\left|z-\varrho_{\mu}\right| \leq b,\left|\arg \left(z-\varrho_{\mu}-\theta\right)\right| \geq \beta$.

Now, observe that $\theta$ can be changed within the interval $\left(\theta^{-}, \theta^{+}\right)$, where $\theta^{-}$(resp. $\theta^{+}$) is the greatest (resp. smallest) singular direction less (resp. greater) than $\theta$. Also $\beta$ can be chosen arbitrarily small positive. Thus, restriction of $G_{\theta}$ to a small left (resp. right) tubular neighbourhood of $\varrho_{\mu}+l_{\theta}$ extends to a holomorphic function defined on $\theta^{-}<\arg \left(z-\varrho_{\mu}\right) \leq 0$ (resp. $\left.0 \leq \arg \left(z-\varrho_{1}\right)<\theta^{+}\right)$. The extension of $G_{\theta}$ obtained this way also satisfies (19) for $d \leq\left|z-\varrho_{\mu}\right| \leq b$ and (18) for $\left|z-\varrho_{\mu}\right| \geq b$.

Thus, by Lemma 3 and the results of Section 1, we get

$$
\frac{1}{2 \pi i} b\left(G_{\theta}\right)=\sum_{\mu=1}^{m} S_{\theta}^{\mu}
$$

where $S_{\theta}^{\mu} \in L_{(0)}^{* \prime}\left(\varrho_{\mu}+\bar{l}_{\theta}\right)$ with $*$ given by (10). So, the solution $w$ of (13) is given by $w(s)=\sum_{\mu=1}^{m} \mathcal{T} S_{\theta}^{\mu}(s)$ for $s \in \Omega_{0}^{\theta}$, and $u(x)=w\left(e^{1-1 / x}\right)$ is defined only for $x \in \frac{1}{2} B\left(e^{i \theta} ; 1\right)$. However, the estimate (17) gives an additional information about $S_{\theta}^{\mu}, \mu=1, \ldots, m$. Namely, changing $\theta$ within $\left(\theta^{-}, \theta^{+}\right)$, we note that the restriction of $S_{\theta}^{\mu}$ to an open ray $\varrho_{\mu}+l_{\theta}$ is analytic, and extends holomorphically to a function $\Psi^{\mu}$ defined in a sector $S\left(\varrho_{\mu} ;\left(\theta^{-}, \theta^{+}\right)\right)$. To estimate $\Psi^{\mu}$ put (with $k=k_{\mu}$ )

$$
\begin{aligned}
& F^{\mu}(\alpha, z)=\frac{A(\alpha, z) \Phi(z-\alpha)\left(z-\varrho_{\mu}-\alpha\right)^{k}}{Q(z) Q(z-\alpha)} \\
& \quad \text { for } \alpha \in l_{\theta^{\prime}}, z \notin \bigcup_{\nu=1}^{m}\left(\varrho_{\nu}+l_{\theta^{\prime}}\right), \theta^{-}<\theta^{\prime}<\theta^{+},
\end{aligned}
$$

where $A(\alpha, z)=A_{\theta^{\prime}}(\alpha, z)$ for $(\alpha, z)$ as above, $\theta^{-}<\theta^{\prime}<\theta^{+}$. Since 
$\left.b(\Phi / Q)\right|_{\varrho_{\mu}+l_{\theta}}=0,(16)$ implies that for $\gamma \in S\left(0 ;\left(\theta^{-}, \theta^{+}\right)\right)$,

$$
\begin{aligned}
\Psi^{\mu}\left(\varrho_{\mu}+\gamma\right) & =\frac{1}{2 \pi i}\left(G_{\widetilde{\theta}^{-}}\left(\varrho_{\mu}+\gamma\right)-G_{\widetilde{\theta}^{+}}\left(\varrho_{\mu}+\gamma\right)\right) \\
& =\frac{1}{2 \pi i} \int_{l_{\tilde{\theta}^{-}}-l_{\tilde{\theta}^{+}}} \frac{F^{\mu}\left(\alpha, \varrho_{\mu}+\gamma\right)}{(\gamma-\alpha)^{k}} e^{-\alpha} d \alpha
\end{aligned}
$$

where $\theta^{-}<\widetilde{\theta}^{-}<\arg \gamma<\widetilde{\theta}^{+}<\theta^{+}$. So for $\gamma$ with $\theta^{-}<\arg \gamma<\theta^{+}$,

$$
\Psi^{\mu}\left(\varrho_{\mu}+\gamma\right)=\widetilde{\Psi}^{\mu}(\gamma) e^{-\gamma}, \quad \text { where } \widetilde{\Psi}^{\mu}(\gamma)=\left.\sum_{l=0}^{k_{\mu}-1} C_{l} \frac{\partial^{l}}{\partial \alpha^{l}} F^{\mu}\left(\alpha, \varrho_{\mu}+\gamma\right)\right|_{\alpha=\gamma}
$$

with some constants $C_{l}, l=0, \ldots, k_{\mu}-1(\mu=1, \ldots, m)$. Observe that (17), (19), and the Cauchy formula imply that for any $\theta^{-}<\widetilde{\theta}^{-}<\widetilde{\theta}^{+}<\theta^{+}$ and $r^{\prime}<r,\left|\widetilde{\Psi}^{\mu}(\gamma)\right|$ can be estimated by the right hand side of (11) for $\widetilde{\theta}^{-} \leq \arg \gamma \leq \widetilde{\theta}^{+}$with some $C<\infty$. Since the above holds for any $r^{\prime}<r$, we conclude that $e^{\gamma} S_{\theta}^{\mu} \in L_{\left(-1 / r e^{-i \theta}\right)}^{* \prime}\left(\varrho_{\mu}+\bar{l}_{\theta}\right)$ and so $S_{\theta}^{\mu} \in L_{(\omega)}^{* \prime}\left(\varrho_{\mu}+\bar{l}_{\theta}\right)$ with $\omega=(\cos \theta-1 / r) e^{-i \theta}$. Now, Lemma 2 implies that $w(s)$ is defined for $s \in \widetilde{\mathbb{C}}$ with $s / e \in \Omega_{-1 / r}^{\theta}$ Finally, $u(x)=w\left(e^{1-1 / x}\right)=\sum_{\mu=1}^{m} \mathcal{L}\left(e^{\gamma} S_{\theta}^{\mu}\right)(-1 / x)$ is defined for $x \in(r / 2) B\left(e^{i \theta} ; 1\right)$, and a direct computation shows that $u$ can be written in the form (12) (with $t=1$ ).

5. An example. Let us solve the Cauchy problem for the Euler equation $x^{2} u^{\prime}=u-x, u(1)=u_{0}$. Putting $s(x)=\exp \{1-1 / x\}$ and $w(s)=$ $u(1 /(-\log (s / e)))$ we get $s w^{\prime}-w=1 / \log (s / e), w(1)=u_{0}$. Applying the Mellin transformation (4) with $0<|\theta|<\pi / 2$ and $t=1$ we obtain the equation for $G_{\theta}=\mathcal{M}_{1}^{\theta} w$,

$$
(z-1) G_{\theta}(z)=-u_{0}+\int_{l_{\theta}} \frac{e^{-\alpha}}{z-\alpha} d \alpha .
$$

Its solution is given by

$$
G_{\theta}(z)=\frac{-u_{0}}{z-1}+\frac{1}{z-1} \int_{l_{\theta}} \frac{e^{-\alpha}}{z-\alpha} d \alpha .
$$

Now, we compute the boundary value $S=(2 \pi i)^{-1} b\left(G_{\theta}\right)$ :

$$
S=\left(u_{0}+A\right) \delta_{(1)}+\int_{l_{\theta}} \log (\alpha-1) \frac{d}{d \alpha}\left(e^{-\alpha} \delta_{(\alpha)}\right) d \alpha
$$

with $A=\left(\Gamma^{\prime}(1)-\sum_{j=1}^{\infty} \frac{1}{j ! j}\right) / e$. Thus, the solution $w=\mathcal{T} S$ is given by

$$
w(s)=\left(u_{0}+A\right) s+\log (s / e) \int_{l_{\theta}} \log (\alpha-1) s^{\alpha} e^{-\alpha} d \alpha \quad \text { for } s / e \in \Omega_{0}^{\theta} .
$$


Finally, $u(x)=w\left(e^{1-1 / x}\right)$ is given by

$$
u(x)=\left(u_{0}+A\right) e^{1-1 / x}-\frac{1}{x} \int_{l_{\theta}} \log (\alpha-1) e^{-\alpha / x} d \alpha \quad \text { for } \operatorname{Re}\left(e^{i \theta} / x\right)>0,
$$

which gives

$$
u(x)=\left[u_{0} e-\sum_{j=1}^{\infty} \frac{1}{j ! j}+\sum_{j=1}^{\infty} \frac{1}{j ! j} \frac{1}{x^{j}}-\log x\right] e^{-1 / x} \quad \text { for } x \in \widetilde{\mathbb{C}} .
$$

\section{References}

[B] B. L. J. Braaksma, Multisummability and Stokes multipliers of linear meromorphic differential equations, J. Differential Equations 92 (1991), 45-75.

[CL] A. E. Coddington and N. Levinson, Theory of Ordinary Differential Equations, McGraw-Hill, New York, 1955.

[E] J. Ecalle, Introduction à l'accélération et à ses applications, Travaux en Cours, Hermann, 1993.

[K] H. Komatsu, Ultradistributions, I. Structure theorems and a characterization, J. Fac. Sci. Univ. Tokyo 20 (1973), 25-105.

[Ł1] G. Łysik, On extendible ultradistributions, Bull. Polish Acad. Sci. Math. 43 (1995), 29-40.

[Ł2] - Laplace ultradistributions on a half line and a strong quasi-analyticity principle, Ann. Polon. Math. 63 (1996), 13-33.

[Ł3] —, The Mellin transformation of strongly increasing functions, J. Math. Sci. Univ. Tokyo 6 (1999), 49-86.

[M] B. Malgrange, Sommation des séries divergentes, Exposition. Math. 13 (1995), 163-222.

[T] J. C. Tougeron, Gevrey expansions and applications, preprint, Univ. of Toronto, 1991.

[Z] B. Ziemian, Generalized analytic functions with applications to singular ordinary and partial differential equations, Dissertationes Math. 354 (1996).

Institute of Mathematics

Polish Academy of Sciences

P.O. Box 137

Śniadeckich 8

00-950 Warszawa, Poland

E-mail: lysik@impan.gov.pl 